\section{$\underset{\substack{\text { hommes } \\ \text { \& migrations }}}{ }$}

\section{Hommes \& migrations}

Revue française de référence sur les dynamiques

migratoires

1308 | 2014

Les Paris des migrants

\title{
Paris, capitale des exils littéraires
}

\section{Mustapha Harzoune}

\section{(2) OpenEdition \\ Journals}

\section{Édition électronique}

URL : http://journals.openedition.org/hommesmigrations/3019

DOI : 10.4000/hommesmigrations.3019

ISSN : 2262-3353

\section{Éditeur}

Musée national de l'histoire de l'immigration

\section{Édition imprimée}

Date de publication : 1 octobre 2014

Pagination : 161-167

ISBN : 978-2-919040-29-2

ISSN : $1142-852 X$

\section{Référence électronique}

Mustapha Harzoune, «Paris, capitale des exils littéraires », Hommes \& migrations [En ligne], 1308 | 2014, mis en ligne le 01 octobre 2017, consulté le 30 avril 2019. URL : http:// journals.openedition.org/hommesmigrations/3019; DOI : 10.4000/hommesmigrations.3019 


\title{
PARIS, CAPITALE DES EXILS LITTÉRAIRES
}

par MUSTAPHA HARZOUNE

\author{
Le 13 octobre 2014, à la BNF, Jake Lamar, Nancy Huston et \\ Elisabeth Niggemann étaient invités pour une rencontre autour \\ de "Paris, capitale des exils littéraires : entre ici et ailleurs". \\ Exil choisi et plutôt heureux pour les deux premiers, \\ exil contraint pour d'autres, car Paris fut aussi une ville refuge \\ pour les persécutés du monde, écrivains ou non.
}

Pour la seule seconde moitié du XX $\mathrm{X}^{\mathrm{e}}$ siècle, Paris a hébergé des dissidents des pays de l'Est, des exilés, chiliens ou argentins, des intellectuels algériens des années 1990 et tant d'autres... Paris reste une ville refuge même si, depuis des années, les politiques d'asile devenant plus restrictives, la fragilité du demandeur d'asile ne fait qu'augmenter. Le 13 octobre dernier, au Sommet du livre ${ }^{1}$, Nancy Huston le rappelait: "Nous savons qu'il y a des gens dans cette situation aujourd'hui en France, et qui peuvent se trouver complètement exaspérés, désespérés par ces 'maisons qui rendent fous' que sont les diverses formes de l'administration française." Reprenant la distinction d'Herta Müller, prix Nobel de littérature 2009, entre exil choisi et exil subi, Élisabeth Niggemann souligne: "Il y a une grande différence quand vous ne pouvez pas rentrer." C'est ce quécrivait Klaus Mann dans Le Tournant: "Peut-on dire que nous étions des émigrés volontaires? Pas tout à fait. Nous ne pouvions pas rentrer. Le dégoût nous aurait tués, le dégoût de notre propre indignité et des ignominies commises autour de nous. L'air du III Reich était, pour certains poumons, irrespirable. Dans notre patrie, la mort par asphyxie nous guettait. C'était une bonne raison, une raison véritablement impérative, de nous en tenir éloignés!" 


\section{Paris, capitale d'une "autre Allemagne"}

Entre 1933 et 1940, plusieurs milliers d'Allemands ont fui le régime nazi pour y chercher refuge. Elisabeth Niggemann, directrice de la Deutsche Nationalbibliothek, détient justement les archives des Allemands exilés entre 1933 à 1945 réparties en deux collections, l'une à Leipzig consacrée aux exils littéraires, l'autre à Francfort-sur-le-Main sur les exilés allemands. Un fonds unique qui a servi à l'organisation de l'exposition L'Art en exil visible sur le site de la Bibliothèque.

Les premiers exilés arrivent en 1933. Parmi eux il y a Walter Benjamin, Hannah Arendt et Klaus Mann, qui quitte l'Allemagne le 13 mars 1933. Déchu de sa nationalité un an plus tard, il fait de son bannissement une marque d'“honneur", malgré les bonnes consciences croisées à Paris, pour qui "cela ne se fait pas. Une personne convenable reste aux côtés de son pays quel que soit celui qui le gouverne". Malgré aussi la culpabilité de l'exil : "Mal à laise, oui, voilà comment on se sentait lorsqu'on pensait au pays. Cette pensée nous venait la nuit, comme elle était venue à l'émigré Heinrich Heine, et il arrivait que, comme lui, elle nous arrachât parfois au sommeil. Elle était chargée de douleur et d'angoisse, cette pensée nocturne. Elle nous rappelait les horreurs qui, chaque jour, devenaient événement dans notre patrie lointaine - et les autres, celles qui allaient encore venir. Ce seraient les plus terribles. Une misère et une destruction d'une étendue jusque-là inconnue s'annonçaient; nous le savions et nous ne pouvions nous empêcher d'y penser pendant nos nuits sans sommeil. Durant le jour, nous tâchions bien d'informer le monde de ce que nous pressentions et de ce que nous savions. Mais personne ne nous écoutait. Nous étions des émigrés." Comme ces intellectuels algériens des années 1990 qui n'eurent de cesse de prévenir du danger de l'islamisme politique et dont sera négligée l'"expérience (...) la seule chose que nous, les proscrits, nous avons à vous offrir" (Klaus Mann).
20000 exilés débarquent en France et spécialement à Paris durant cette période. Juifs allemands, communistes, sociaux-démocrates, intellectuels fuient l'Allemagne pour des raisons politiques. Beaucoup se retrouvent sans ressources, ne parlant pas le français, et ce, quelle qu'ait été leur condition ou profession. À Paris, l'exil allemand se poursuivra jusqu'en 1940. Un séjour long pour certains, bref pour d'autres. L'arrivée des soldats de la Wehrmacht sonne l'heure d'un autre départ.

Avec l'occupation nazie, des réfugiés allemands à l'instar de Heinrich Mann et de Walter Benjamin rejoignent le sud du pays pour gagner l'Espagne ou les États-Unis. Certains tentent de survivre grâce à l'aide d’amis français. Beaucoup sont déportés en Allemagne, d'autres, antinazis de la première heure, devenus "étrangers ennemis", sont internés en France, comme Max Ernst dans le camp des Milles près d'Aix-en-Provence, Walter Benjamin dans celui de Vernuche près de Nevers ou Hannah Arendt dans le camp de Gurs. Parmi ceux qui échappèrent aux balles des tueurs du "baratineur à la petite moustache", combien se suicidèrent? Des vies difficiles, "courtes et tristes", dont témoignent journaux intimes et écrits conservés à la Deutsche Nationalbibliothek.

Ainsi, entre 1933 et 1940, Paris devint la capitale d'une "autre Allemagne". Grâce à ses artistes, écrivains et intellectuels, l'Allemagne de Goethe et de Hölderlin continuait, en exil, de vivre. Les Josef Breitenbach, Bertolt Brecht, Helene Weigel, Max Ernst, Klaus Mann, Heinrich Mann... participaient aussi, malgré eux, au lustre de Paris, capitale des arts et des lettres

\section{Paris, capitale des arts et des lettres}

Jake Lamar est journaliste et auteur afro-américain. Né en 1961 dans le Bronx à New York, il est âgé de 28 ans quand il reçoit la commande d'une autobiographie. Ce sera Bourgeois Blues, dont la tra- 
duction, Confessions d'un fils modèle, sera publiée à Paris en 2009 (chez Payot et rééditée chez Rivages/ Poche en 2012). Gratifié d'un prix, il décide de se consacrer à l'écriture et de satisfaire un vieux rêve, une "curiosité" à tout le moins : partir pour Paris. En 1993, il s'installe dans le XVIII arrondissement, du côté de Montmartre, où il réside toujours. À 32 ans, Jake Lamar ne parle pas un mot de français. Comme il pense repartir au bout d'un an, il ne s'embarrasse pas à apprendre la langue du pays d'accueil. Cela durera quatre ans, le temps de réaliser qu'une bonne part de son avenir est parisien et qu'il est temps de prendre "au sérieux l'effort d'apprendre la langue".

Jake Lamar entretient pourtant avec Paris une déjà longue histoire, une histoire qui le ramène à d'autres écrivains américains de la capitale française. “À l'âge de 12, 13 ans, j'ai lu le premier roman de James Baldwin (Go Tell it On the Mountain, 1953). C'est un livre qui m'a frappé. J'étais un enfant dans une famille difficile, dans le Bronx. Dans ce livre, Baldwin a écrit sur son enfance dans une famille difficile à Harlem. Après ma lecture, j’ai demandé à mon professeur qui était cet auteur, il m'a dit 'Il habite à Paris'. C'était une idée très exotique pour moi, quelqu'un avec des racines comme ça qui s'était installé à Paris... Plus tard au moment où j'ai pensé que je serais peut-être écrivain, j’ai aussi eu l'idée qu'un jour j'habiterais à Paris."

D’autres écrivains américains guideront les pas de Lamar vers Paris. Après Baldwin, il cite Richard Wright, puis Francis Scott Fitzgerald, Ernest Hemingway, Gertrude Stein, Henry Miller... "La liste est très longue." C'est le Paris capitale des arts et des lettres qui exerça une "attirance" sur Jake Lamar, d'autant plus que "la France est un pays où les arts en général, l'écriture en particulier, sont appréciés. Aux États-Unis, ce n'est pas le métier lui-même qui est respecté, c'est l'idée de succès, l'idée d'être un écrivain riche et célèbre. Ce n'est pas simplement le métier. Quand vous êtes un écrivain comme moi qui ai publié sept livres mais pas un best-seller, les Américains se demandent pourquoi je continue. Ce riest pas le cas en France, où je suis apprécié comme quelqu'un de dévoué à ce métier atypique."

Nancy Huston est née à Calgary au Canada. Anglophone, elle a grandi, hormis une brève parenthèse allemande, en Alberta puis dans le New Hampshire, et étudié à Cambridge (Massachussetts) puis à New York. Elle arrive à Paris en 1973, à

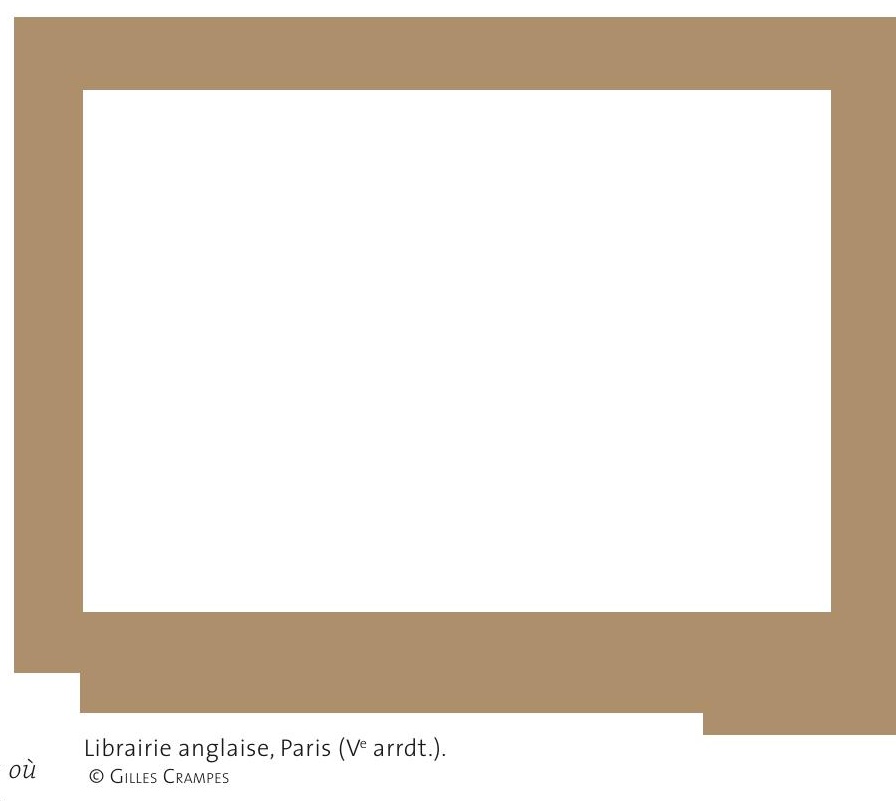

20 ans, pour y finir ses études. Romancière et essayiste bilingue, elle est l'auteure d'une quarantaine de livres (romans, essais, théâtre, correspondance, jeunesse...). En 2014, dans Bad Girl (Actes Sud), elle raconte "comment quand on est né dans le Canada anglophone on devient une romancière parisienne".

"Être une Parisienne ou une Française de souche ne me fait pas le moins du monde envie. En revanche, être artiste en exil à Paris, c'est peut-être une des situations de vie humaine les plus enviables, les plus merveilleuses. Parce qu'ils se rencontrent entre eux, les 
artistes ne sont ni d'ici, ni de là-bas, ils sont libres pour travailler, rêvasser, créer... et n'ont pas trop à penser à leurs antécédents." Pour les artistes étrangers, "Paris peut être une sorte de paradis sur terre". "Ensuite, s'il faut montrer patte blanche, commencer à se comporter comme un 'bon petit FranNancy Huston : "Je me sens chez moi parmi les étrangers, c'est devenu mon identification principale. Je suis et serai toujours une étrangère, partout." çais', obéir, remplir tous les formulaires..., on peut perdre cette belle sérénité qu’on avait comme artiste."

Pour illustrer son parcours d'exilée, elle reprend les "trois phases de l'habituation à l'exil" selon Tzvetan Todorov : "leuphorie, la dysphorie et le désespoir serein". "L'euphorie, c'est évidemment la première phase, quand on arrive et et commence à bien parler la langue... Mais ce n'est pas seulement une question de maîtrise de la langue. On absorbe comme une éponge tous les codes, toutes les références... et c'est totalement euphorisant. J'ai vécu ça la première dizaine d'années en France, où j’allais dans les séminaires de Jacques Lacan, de Roland Barthes, je lisais Foucault, Kristeva, les féministes, Beauvoir... J'ingurgitais tout ça. Je me racontais que jétais née en fait à 20 ans, que j'étais une brillante intello parisienne et que je n'avais pas d'enfance, pas de langue maternelle, pas de pays natal."

\section{Paris, Ville-monde}

Quand il arrive à Paris, Jake Lamar découvre le XVIII arrondissement, "une des grandes surprises pour moi était à quel point Paris est une ville multiculturelle. Quand je suis arrivé en 1993, j'ai découvert le XVIII arrondissement et j'y ai été très à l'aise", aussi à l'aise que dans ces quartiers mélangés, mixtes de New York qu'il affectionne. "Quand j'ai écrit mon premier livre qui se déroule à Paris (Rendez-vous dans le XVIII', Rivages, 2007), le décor était le XVIIIe. J'ai attendu environ sept ans avant de me sentir prêt à écrire sur Paris, décrire le Paris que je connaissais, ce Paris que je n'ai pas vu souvent capté dans la littérature américaine, le Paris multiculturel."

Nancy Huston décrit une autre trajectoire. Elle a récemment délaissé le $\mathrm{V}^{\mathrm{e}}$ arrondissement, celui du Quartier latin, pour le XXe : "Ce choix de quartier reflète l'évolution de mon sentiment envers la France, je ne suis plus à l'aise à jouer les intellos français au Quartier latin. Je me sens chez moi parmi les étrangers, c'est devenu mon identification principale. Je suis et serai toujours une étrangère, partout. Je me suis toujours sentie une outsider - c'est le titre de la nouvelle traduction de L'Étranger de Camus." C'est ici que l'exil parisien de Nancy Huston semble atteindre la deuxième des "trois phases de l'habituation à l'exil" de Tzvetan Todorov, celui de la dysphorie: "La dysphorie est venue au début de la trentaine, liée probablement au fait d'être devenue mère et d'avoir eu beaucoup de mal à parler l'anglais avec mes enfants, d'avoir trouvé cela douloureux. Des sortes de souvenirs de l'enfance ont commencé à affleurer et vers 32, 33 ans, j’ai fait une crise physique et psychique qui m'a amenée peu à peu à ressusciter ma langue maternelle et à m'en servir pour écrire pour la première fois. J'avais déjà publié une dizaine de livres en français et je me suis mise à écrire en anglais Cantique des plaines, une sorte de retour imaginaire à ma province. Ça, cétait la dysphorie, et en fait je dirais que je suis toujours dans cette phase, qu'elle va croissante et que je pense ne jamais atteindre au désespoir serein."

\section{Écrire en exil}

Parmi les livres déjà parus de Jake Lamar, deux sont des romans dont l'action se situe à Paris (Rendez-vous dans le XVIII e et Les Fantômes de SaintMichel). Chez l'auteur américain, l'exil marque de son empreinte les sujets, les thèmes et les cadres qu'il aborde : "C'est vraiment clair avec mes deux derniers ouvrages, la pièce de théâtre [Brothers in Exile] que je n'aurais jamais écrite sans avoir eu cette expérience d'expatrié et mon nouveau roman, Postérité [Payot-Rivages], dont une grande partie se déroule aux Pays-Bas." Depuis qu'il vit à Paris, lui le citoyen 
américain qui a biberonné à "comment les ÉtatsUnis ont sauvé le monde", se questionne dorénavant sur la façon dont la Seconde Guerre mondiale a été vécue en Europe. Postérité raconte l'histoire d'une femme peintre hollandaise qui a vécu l'occupation nazie. "Dans mon immeuble, il y avait une femme qui était connue comme l'ange de Montmartre, elle était dans la Résistance. Quand j'ai entendu les histoires de dénonciation des voisins..." De même, avoir lu aux États-Unis Le Journal d'Anne Franck, "c'est une chose, reconnaît Jake Lamar, mais être dans l'appartement où elle était cachée à Amsterdam, c'est une expérience complètement différente... et je pense que c'est ça qui m'a inspiré pour écrire ce livre. Sans mes années en Europe, je n'aurais jamais écrit cette histoire".

La pièce Brothers in Exile met en scène James Baldwin, Richard Wright et Chester Himes. Comme nul n'est prophète en son pays, "une des grandes surprises pour moi quand je suis arrivé à Paris a été de découvrir Chester Himes. Chester Himes reste très peu connu aux États-Unis, même les experts de la littérature afro-américaine ne connaissent pas très bien son ceuvre. C'est à Paris que les gens mont dit que Chester Himes est un grand auteur. Et c'est comme si je découvrais un grand-père dont j'ignorais l'existence, un ancêtre secret. Je me suis senti un vrai rapport, en tant qu'écrivain, avec l'œuvre de Chester Himes qui est un auteur troublant pour les Américains, par la façon dont il aborde les questions raciales, avec une attitude très cynique vis-à-vis des Noirs et des Blancs".

La pièce de Jake Lamar s'inspire d'une "engueulade" historique : la confrontation aux Deux-Magots, à Saint-Germain-des-Prés, en 1953, entre Baldwin et Wright. Cela a duré "des heures et des heures. Chester Himes était à la table. Chacun des trois a écrit une version très différente de ce qui s'est déroulé ce soir-lă". Pour Richard Wright, l'exil parisien relevait de l'obligation, "il ne pouvait pas rentrer aux ÉtatsUnis à cause de la menace du maccarthysme, il était toujours menacé, toujours soupçonné d'être communiste bien qu'il ait rejeté le Parti communiste. Mais il ne pouvait pas rentrer, c'était vraiment l'exil pour lui". Pour James Baldwin, l'exil était plus "littéraire" : "Il ne pouvait pas devenir écrivain sans quitter les ÉtatsUnis. Il était si opprimé par le racisme quotidien, même à New York, qu'il ne pouvait pas devenir écrivain, comme il l'a dit lui-même (et je suis d'accord) sans quitter les États-Unis. Mais il est rentré assez souvent, tout au long de sa vie." Quant à Chester Himes, "il nétait heureux nulle part, et à la fin de sa vie, il déclarait qu'il n'avait pas trouvé sa place. Il n'était à l'aise ni aux États-Unis, ni en France, ni en Espagne où il est mort. [Toute sa vie] il a cherché un endroit qu'il n'a pas trouvé."

Très à l'aise dans "ses" deux villes, Paris et New York, Jake Lamar, lui, "hésite à utiliser le mot exil": "Je suis venu par curiosité, je suis resté parce que jétais heureux. Je n'envisage pas un retour pour vivre aux États-Unis. Mais mon choix de rester à Paris n'a jamais été un choix contre les États-Unis."

S'appuyant sur ses "maîtres à penser", ses "maîtres à écrire", Nancy Huston propose une grille des démarches adoptées par des écrivains en exil, en France ou à Paris : "Il y a la démarche anorexique, de Samuel Beckett, qui a su profiter de son statut d'alien pour se comporter en alien. Après l'épisode important de la Résistance, auquel il a participé non par conviction politique mais par conviction humaine, tout simplement parce que la vie de certains des siens, de ses amis, était
Jake Lamar : "Je suis venu par curiosité, je suis resté parce que j'étais heureux. Je n'envisage pas un retour pour vivre aux États-Unis. Mais mon choix de rester à Paris n'a jamais été un choix contre les États-Unis." en danger, Beckett n'a plus pris part au débat politique français, il ne s'est intégréà rien du tout. Il a inventé un univers sans pays, sans lieu, sans langue particulière. "Deuxième possibilité, la démarche boulimique de Romain Gary. Gary, sachant que l'identité est une construction, décide d'avaler les histoires des autres, d'être tout le monde, il est un ogre, il peut écrire sur les hommes, les femmes, les enfants de n'importe quel continent, n'importe quelle condition, n'importe quel siècle. Mais, d'exil en exil, à force de se fuir, il finit par se mettre une balle dans la tête. Et je trouve important que le dernier roman de Romain Gary, Les Cerfs- 
Volants, ait été la tentative de réconcilier ses deux mondes : celui qui a humilié sa mère, la Pologne, et celui qui l'a fait homme, la France de De Gaulle.

"On peut aussi, troisième possibilité, tranquillement et à jamais, écrire dans sa langue d'adoption des romans qui portent sur son pays natal, c'est par exemple le cas d'Andreï Makine, de Michel del Castillo et d'autres. Ou enfin, on peut vaciller comme Jake et moi : tantôt làbas, tantôt ici, tantôt dans des voyages entre les deux, l'articulation des deux, parler de l'exil à l'intérieur des livres mêmes. J'ai fait une tentative un peu étrange dans mon dernier roman, Danse noire, de parler du bilinguisme, enfin de jouer avec le bilinguisme."

\section{Regards croisés}

Si Jake Lamar s'est tout de suite senti à l'aise, "heureux" même, à Paris, il ne cache pas son aversion pour certaines "spécificités" françaises comme "les grèves par exemple". "Je ne suis pas un citoyen français... Une amie a noté que, lorsqu'on me demande d'où je viens, je dis que je suis new-yorkais avant de dire que je suis américain, et je pense que je suis vraiment identifié avec ma ville natale, et dans mon idée de la culture du business, je reste new-yorkais, et je pense que souvent mes frustrations en France sont liées à la culture du business : les grèves, les contrats..."

L'exil est aussi liberté. "Je suis bien dans ce sentiment de déplacement, dit Nancy Huston. Je trouve que c'est bien pour écrire. Il ne faut pas coïncider, il faut toujours avoir un recul, une distance, un regard. Si on fait un avec son pays, avec sa langue, etc., on est trop près. L'exil est de ce point de vue très propice à la création."

Jake Lamar le reconnaît : "D'une certaine façon, je me sens libéré de certaines préoccupations américaines, surtout des préoccupations raciales. Je ne dis pas qu'il n'y a pas de racisme en France, le racisme est une caractéristique humaine, cela existe dans le cour des gens, partout. Mais il y a un paradoxe entre les États-Unis et la France. Aux États-Unis, grâce au mouvement des droits civiques, grâce à une importante présence des Noirs depuis le XVII siècle, même si c'étaient des esclaves, mais en tout cas les Noirs étaient là depuis le début du pays. Aux États-Unis, il y a beaucoup plus d'opportunités pour les Noirs. On voit beaucoup plus d'avocats noirs, de médecins noirs, d'hommes d'affaires noirs, beaucoup plus d'étudiants dans les grandes écoles qui sont noirs... Mais en même temps, il y a toujours une méfiance entre les groupes, une sorte de chacun chez soi. En France, c'est le contraire, il y a plus de racisme 'institutionnel', c'est-à-dire qu'il est beaucoup plus rare de voir des étudiants noirs dans des grandes écoles, les avocats, les médecins, les hommes d'affaires noirs qui ont réussi sont plus rares en France. Mais en même temps, et c'est le paradoxe, je pense que dans leur vie quotidienne les gens sont beaucoup plus à l'aise les uns avec les autres. C'est beaucoup plus commun en France de voir des couples mixtes, des groupes d'amis mixtes. Moi j'ai voyagé en France jusque dans les petits villages où je n'ai jamais ressenti une méfiance. Le problème en terme de racisme, en France comme aux États-Unis, arrive avec la police. J'ai été contrôlé en France plusieurs fois, sans aucune raison évidente, saufla couleur de ma peau. Mais dès que le flic voit ma nationalité ou ma carte de résidence, au moment où je commence à parler et qu'il entend mon accent, il n'y a aucun problème. Ce n'est pas le cas pour mes amis africains, maghrébins, antillais, mais ça c'est un truc dans les sociétés, jétais aussi surveillé à New York par les flics, cela est lié au fait d'être un homme noir dans le monde occidental."

Si le provincial qui débarque à Paris est massivement en situation de stress et ne rêve que de rejoindre ses verts pâturages, son cadre de vie douillet et chaleureux, les bonnes et grandes tablées entre amis, l'écrivain new-yorkais vit la capitale parisienne comme un havre de paix comparé au "stress, au bruit, à la foule, à l'air de menace, au subway" de la Grosse Pomme. "New York, cétait formidable quand jétais jeune, plein d'énergie, mais maintenant je me sens un peu vieux pour New York, je trouve ça fatigant... J'adore New York mais après trois jours, je suis prêt à rentrer à Paris, et chaque fois, à mon retour, je suis très soulagé d'être à Paris." 
Nancy Huston a acquis la nationalité française en épousant un Bulgare. Citoyenne française, elle vote depuis 1981 et, tout en louant la capacité de la culture française à intégrer des voix étrangères et à traduire aussi la littérature étrangère, elle dit pourtant se sentir "de moins en moins française". "Les aspects de la société et de la culture françaises qui me plaisaient le plus à 20 ans sont à 60 ans ceux qui me plaisent le moins. La volubilité en particulier. Les Français parlent comme un livre. Pendant de longues années, j'ai été portée, transportée par leur verbosité, leur don, leur passion du verbe, et maintenant presque chaque jour, à n'importe quelle heure du jour, dès que jouvre la radio, j'ai envie de la refermer. Je ne supporte plus d'entendre se déployer toutes ces certitudes, ces suffisances ; j'ai besoin de silence pour réfléchir." Pas sûr qu'il faille être d'origine étrangère pour ressentir les mêmes émotions et besoins...

\section{Paris, "Tout-Monde"}

Sans comparaison avec l'exil d'un Chester Himes, Nancy Huston reconnait ne pas pouvoir rentrer au Canada ou dans le New Hampshire. Après quarante et un ans à Paris, elle qui dit ignorer ce que serait un "chez-moi" ne s'y sent toujours pas chez elle. "Depuis plusieurs années je partage ma vie avec un peintre suisse, du coup je vis maintenant à cheval entre Paris Gambetta et un minuscule village du canton de Fribourg! Et je préserve, de plus, un lien fort au Berry, région française qui a inspiré deux de mes romans et où est né un de mes enfants... Venant après la NouvelleAngleterre et New York, je vois que j’ai vécu en fait plusieurs exils - et pourtant, le pays natal reste primordial dans le coeur. Quand je parle de coeur, je parle du noyau de l'être, parce que l'enfance, pour tout le monde, est quelque chose de nature différente du reste de la vie. Probablement qu'on ne peut pas être un bon poète ou un grand romancier si on oublie totalement ce qu'on a vécu, absorbé, senti, goûté, entendu pendant ces années-là, littéralement formatrices. En ce sens, je resterai toujours canadienne."
"En fait, je trouve magnifique d'être dans ces déplacements dont parlait Julia Kristeva ${ }^{2}$, c'est-à-dire dans la perte du naturel. Elle disait 'quand on apprend une autre langue, on s'éloigne de la source', comme un détachement d'avec tout ce qui paraissait évident. Mais c'est bien! parce qu'il s'agit des fausses évidences. De ce point de vue, l'apprentissage d'une ou de plusieurs langues étrangères est certainement facteur de civilisation. Mais cela peut être aussi facteur de détresse existentielle. C'est un équilibre à chercher, un équilibre que l'on ne trouve peut-être jamais. Je pense en effet n'avoir jamais connu un sentiment d'adhésion, de patriotisme, ou alors par procuration. Par exemple, en regardant Le Bal d'Ettore Scola, j'ai été bouleversée de sentir l'effet de l'Occupation allemande sur cette salle de bal à Paris, alors que ce n'est pas du tout mon histoire. Et je l'ai été souvent aussi par des événements liés soit à la guerre d'Algérie, soit à la Deuxième Guerre mondiale. Plusieurs de mes romans se déroulent dans les quartiers juifs de New York ou de Paris (j’ai vécu vingt-cinq ans dans le Marais)... Je pense que j’avais tellement envie d'échapper à ma propre histoire que je suis devenue infiniment ouverte à l'histoire et à la souffrance des autres." 